\title{
Elucidation of a Novel Mechanism of Presepsin Production by Monocyte/macrophage Phagocytosis of Neutrophil Extracellular Traps
}

Akishige lkegame ( $\square$ ikegame-a@chs.pref.kagawa.jp )

Kagawa Prefectural University of Health Sciences

Akihiro Kondo

Kagawa Prefectural University of Health Sciences

Ken Kitaguchi

Kagawa Prefectural University of Health Sciences

Kanami Sasa

Kagawa Prefectural University of Health Sciences

Masashi Miyoshi

Tokushima University Hospital

Research Article

Keywords:

Posted Date: February 24th, 2022

DOI: https://doi.org/10.21203/rs.3.rs-1346142/v1

License: (c) (1) This work is licensed under a Creative Commons Attribution 4.0 International License.

Read Full License 


\section{Abstract}

Presepsin, a biomarker discovered in Japan, has been clinically applied as a diagnostic aid for sepsis. Recently, however, it has been reported that presepsin levels are elevated in patients with severe systemic lupus erythematosus without infection, suggesting the existence of a production mechanism that does not involve bacterial phagocytosis. In this study, we aimed to elucidate the mechanism of presepsin production without bacterial phagocytosis and explore the clinical significance of presepsin. Neutrophil extracellular traps (NETs) were induced by Escherichia coli and phorbol myristate acetate (PMA) in neutrophils isolated from the peripheral blood of healthy subjects. NET induction alone did not increase presepsin levels, but co-culturing with monocytes significantly increased them. The addition of a NET formation inhibitor also suppressed presepsin levels, suggesting that presepsin production is greatly influenced by monocyte phagocytosis of NETs. Phagocytosis of NETs by THP-1 and U937 cells, which was induced by CD14 expression, also increased presepsin levels. This study suggests that presepsin can be used to assess the severity of inflammatory diseases, such as autoimmune diseases, and monitor treatment effects.

\section{Introduction}

Sepsis is a common condition associated with unacceptably high mortality and long-term morbidity in many survivors ${ }^{[1,2]}$. Therefore, early diagnosis is important for treating sepsis, and because it has been reported to have a significant impact on the subsequent course of the disease, biomarkers that can be measured rapidly with high specificity are required ${ }^{[3,4]}$.

Cluster of differentiation 14 (CD14) has been described as a monocyte/macrophage differentiation antigen on the surface of myeloid lineages, such as monocytes, macrophages, and dendritic cells (DCs) [5]. This protein plays a crucial role in immune recognition and reactivation of microbial cell wall components in gram-positive and gram-negative bacteria ${ }^{[6]}$. Recently, CD14 has been shown to play a role in the phagocytic clearance of apoptotic cells ${ }^{[7]}$. CD14 isoforms (52-55 kDa) expressed on the surfaces of monocytes and neutrophils, membrane protein (mCD14), are attached to the cell surface by a glycosyl phosphatidylinositol (GPI) anchor, while the other isoform is a serum soluble (48-56 kDa) (sCD14, an acute-phase protein) ${ }^{[8]}$. Soluble CD14 found in human serum has been attributed to the shedding of mCD14 from monocytes, macrophages, and PMNs. Membrane CD14, a receptor for lipopolysaccharide (LPS) on the membrane of mononuclear phagocytes (MPS), binds to LPS-binding protein (LBP) in plasma and transfers it to the cell surface receptor CD14 ${ }^{[9]}$. LPS stimulates human monocyte activation via several intracellular signaling pathways that involve proinflammatory factors ${ }^{[10]}$.

sCD14 plays an important role in mediating immune responses to LPS in CD14-negative endothelial and epithelial cells. During inflammatory stress, sCD14 is cleaved in the plasma to generate a $13 \mathrm{kDa}-\mathrm{N}$ terminal fragment which has been identified as an SCD14 subtype (SCD14-ST; also known as presepsin) $[11-13]$ 
The mechanism of presepsin production is as follows: when phagocytes phagocytose bacteria infecting blood vessels during sepsis, CD14 expressed on the surface of phagocytes is taken up together with the bacteria and is degraded by neutrophil elastase, a proteolytic enzyme, to produce presepsin ${ }^{[14]}$. Therefore, presepsin has a high specificity as a sepsis biomarker. Furthermore, presepsin is a useful biomarker for the early detection of sepsis owing to its short half-life $(4 \mathrm{~h})^{[15-17]}$.

On the other hand, elevated presepsin levels have been reported in patients with systemic lupus erythematosus (SLE) and hemophagocytic syndrome, autoimmune diseases without evidence of infection, causes of which remain unknown ${ }^{[18-20]}$.

In 2004, Brinkmann et al. described neutrophil extracellular traps (NETs), which actively release their DNA into the extracellular space, as a completely new form of neutrophil defense. NETs bind to gram-positive and gram-negative bacteria to differentiate and eliminate virulence factors ${ }^{[21]}$. In sepsis, neutrophils and monocytes/macrophages phagocytose bacteria that invade blood vessels, and NETs release calcium ions stored in the endoplasmic reticulum (ER) into the cytoplasm when cytokines complementarily bind to and activate toll-like receptors (TLRs) and other receptors on neutrophils. An increase in the concentration of calcium ions in the cytoplasm activates protein kinase $C$ (PKC). Activated PKC induces the activation of nicotinamide adenine dinucleotide phosphate (NADPH) oxidase and ROS production [22, 23]. ROS induces transfer of peptidylarginine deiminase 4 (PAD4) to the nucleus, where it changes arginine in the histone tails to citrulline, weakening its binding to DNA by changing its conformation, causing DNA to decondense and be released from the cell ${ }^{[24,25]}$. Neutrophils stimulated by high mobility group protein B1 (HMGB1) released via LPS or dead gram-negative rods mediated activation, release NETs composed of chromatin fibers containing DNA and citrullinated histones within 2-4 h. NETs include more than 30 proteins, including myeloperoxidase (MPO), neutrophil elastase (NE), and HMGB1 $^{[26,27]}$. Extracellular stimulators of NET production have been reported to be induced not only by LPS from gram-negative rods but also by substances such as PMA and monosodium urate crystals ${ }^{[28,}$ 29].

Citrullinated histones released from cells by NETs act as deadly DAMPSs ${ }^{[30]}$ disrupt the vascular endothelium, especially in the microvascular environment, activate the blood coagulation system, and induce septic DIC and deep vein thrombosis ${ }^{[31-33]}$. NETs that have completed their role in vivo are rapidly degraded by DNase I in serum. However, in the blood of patients with SLE, DNase I activity is reduced due to autoantibodies against DNA and histones, which interfere with NET clearance ${ }^{[20,34]}$.

In this study, we aimed to elucidate the cause of high presepsin levels in SLE and hemophagocytic syndrome and demonstrate a novel mechanism of presepsin production by monocytes and macrophages phagocytosing NETs, in addition to the known presepsin production mechanism.

\section{Results}


NET induction by bacterial and PMA stimulation is induced in a concentration-dependent manner.

In the induction of NETs by DH5a stimulation of peripheral blood isolated neutrophils, the ratio of NETs to the amount of DH5a at ODs of 0.01 and 0.1 was not significantly different from that of untreated neutrophils. However, at an OD of 1.0 significant increase in the NET ratio to $12.1 \pm 2.1 \%(p<0.01)$ was observed. DH5a-NETs were defined as NETs that were stimulated by DH5a at a bacterial concentration of OD 1.0. In the induction of NETs by PMA stimulation, $5 \mathrm{nM}$ PMA $(0.6 \pm 0.0 \%)$ did not show a significant difference from untreated neutrophils $(0.7 \pm 0.0 \%)$; however, the NET ratio increased in a concentrationdependent manner with $25 \mathrm{nM}$ PMA $(25.6 \pm 2.3 \%)$ or more $(p<0.01)$. The NET ratio increased in a PMA concentration-dependent manner at PMA concentrations of $50 \mathrm{nM}(28.7 \pm 2.4)$ and $100 \mathrm{nM}(33.1 \pm 2.8 \%)$ (Fig. 1A, B). The optimum PMA concentration was determined to be $50 \mathrm{nM}$ (PMA-NETs) because of the slow increase in the NET ratio with 100 nM PMA.

\section{Two types of NETs with different morphologies were identified.}

Two types of NET morphologies were observed when stained using the May-Giemsa staining method, namely NET-type 1, in which the cytoplasm was disrupted, and NET-type 2, in which the nuclear membrane was disrupted and DNA was released outside the cell. Both forms of NETs captured bacteria, and the MPO released extracellularly was positive (Fig. 2A, B). Compared with PMA-NETs, DH5a-NETs showed a higher number of NETs with a large type-ll morphology that released DNA extracellularly and captured bacteria.

\section{NETs express high levels of extracellular CD14, MPO, and NE.}

The Median fluorescence intensity (MFI) values of CD14, MPO, and NE on the cell surface by flow cytometry were $0.7 \pm 0.0 \%, 0.3 \pm 0.0 \%$, and $1.7 \pm 0.5 \%$ for untreated neutrophils and $1.6 \pm 0.2 \%, 2.3 \pm 0.6 \%$, $6.0 \pm 0.6 \%$ for neutrophils stimulated by DH5a, while the NET area was $5.1 \pm 0.9 \%, 13.3 \pm 1.9 \%, 28.1 \pm$ $3.4 \%$, respectively. All three genes were highly expressed in the NET area than in the untreated cells. Specifically, the expression of CD14, the source of presepsin in the NET area, was significantly higher than that in the neutrophils (Fig. 2C).

\section{Evaluation of extracellular citrullinated histones and CD14 in NETs by immunofluorescence imaging.}

Although citrullinated Histone $\mathrm{H} 3$ (Cit-H3) was negative in untreated neutrophils, we found neutrophils that were positive for Cit-H3, which is essential for NET stimulation by DH5a and PMA, revealing the presence of NETs. The extravasated nuclei of these cells stained positively with SYTOX green. The Cit-H3positive NETs were extracellularly positive for MPO and NE (Fig. 2D,E). NETs stimulated by DH5a and PMA showed higher CD14 expression than the untreated neutrophils, consistent with flow cytometry measurements (Fig. 2F).

\section{Evaluation of presepsin production in the induction of NETs.}


After inducing PMA-NETs, we observed the morphological images of monocytes that phagocytosed dead NETs by co-culturing with monocytes (Fig. 3A). NETs and monocytes were co-cultured and stained for CD14, citrullinated histones, and presepsin and evaluated by immunofluorescence imaging. Citrullinated histones were not observed in untreated cells, but DH5a-NETs and PMA-NETs were positive for the same (Fig. 3B). In addition, monocytes phagocytosed NETs with high CD14 expression, and presepsin was produced intracellularly in monocytes (Fig. 3C). Presepsin levels in untreated neutrophils, DH5a-NETs, and PMA-NET-induced NET supernatants were compared. Compared with presepsin levels in untreated neutrophils $(11.8 \pm 2.4 \mathrm{pg} / \mathrm{mL})$, those in DH5a-NETs $(22.5 \pm 0.8 \mathrm{pg} / \mathrm{mL})$ and PMA-NETs $(20.4 \pm 3.4 \mathrm{pg} / \mathrm{mL})$ showed a statistically significant increase but did not show a dramatic difference (Fig. 3D). The induction of NETs alone did not result in significant changes in presepsin levels. However, when monocytes were co-cultured with NETs, presepsin levels in DH5a-NETs $(31.4 \pm 3.4 \mathrm{pg} / \mathrm{mL})$ and PMA-NETs $(92.4 \pm 3.3$ $\mathrm{pg} / \mathrm{mL}$ ) increased substantially (Fig. 3E). Furthermore, the NET ratio increased in a concentrationdependent manner under both DH5a and PMA stimulation. Presepsin levels increased similarly as monocytes phagocytosed NETs (Fig. 3F, G).

\section{Presepsin is produced by monocytes to phagocytose NETs.}

In both DH5a-NETs and PMA-NETs, extracellularly released Cit-H3 was observed, and CD14 was highly expressed. In addition, CD14 and presepsin were found in cells phagocytosed by monocytes (Fig. 3C). Monocytes phagocytose dead NETs and take them into the cells to produce presepsin.

\section{Presepsin levels are lowered by suppressing NETs.}

We examined the effect of diphenyleneiodonium chloride (DPI) on presepsin production under conditions in which NETs were suppressed. Compared to that in PMA-NETs, the NET ratio in DPI (0.1-10 $\mu \mathrm{M})$ pretreatment before PMA stimulation was lower, and NETs were suppressed (Fig. 4A). Furthermore, when the cells were co-cultured with monocytes and phagocytosed by monocytes, presepsin levels lowered in a DPI concentration-dependent manner. In other words, presepsin levels decreased in a concentrationdependent manner when NETs were suppressed (Fig. 4B). Inhibition of NETs by $10 \mu \mathrm{M}$ DPI suppressed both DH5a-NETs and PMA-NETs. Similarly, the presepsin levels after co-culture with monocytes also decreased, and the presepsin level reflected the number of NETs (Fig. 4C, D).

\section{Evaluation of presepsin for NET suppression using cytochalasin D and sivelestat.}

Presepsin levels were evaluated under conditions where the phagocytic activity of monocytes was inhibited by cytochalasin D. In addition, we evaluated presepsin levels using sivelestat, an inhibitor of neutrophil elastase, which is a proteolytic enzyme of monocytes. Presepsin levels in neutrophils cocultured with monocytes (untreated) were $30.7 \pm 7.7 \mathrm{pg} / \mathrm{mL}$; those in PMA-NETs co-cultured with monocytes were $158.7 \pm 5.8 \mathrm{pg} / \mathrm{mL}$; and those under conditions of cytochalasin D-inhibited phagocytosis were $115.7 \pm 4.0 \mathrm{pg} / \mathrm{mL}$. Inhibiting the phagocytic activity of monocytes significantly decreased presepsin levels $(p<0.01)$. In addition, under conditions of neutrophil elastase inhibition by sivelestat, the presepsin 
levels were $121 \pm 19.5 \mathrm{pg} / \mathrm{mL}$, which were significantly lower than those in the PMA-NETs phagocytosed by monocytes $(p<0.05)$ (Fig. 5$)$.

\section{Presepsin is produced by macrophages to phagocytose NETs that express high CD14 levels.}

CD14 expression in U937 and THP-1 cells showed mean fluorescence intensities (MFIs) of 1.3 and 1.5, respectively. Both cell lines showed significant CD14 expression with MFIs of 5.5 and 8.6 after 1a, 25dihydroxyvitamin D3 (VD3) stimulation to induce differentiation into macrophages (Fig. 6A).

Presepsin levels (untreated) in VD3-stimulated U937 and THP-1 cells were 16.8 \pm 2.6 and $15.9 \pm 1.7$ $\mathrm{pg} / \mathrm{mL}$, respectively, while those after co-culture of PMA-NETs with U937 and THP-1 cells increased to $31.2 \pm 2.0$ and $43.5 \pm 1.6 \mathrm{pg} / \mathrm{mL}$, respectively. In addition, the presepsin levels in both cell lines decreased after NETs were suppressed by DPI treatment and the cell lines were co-cultured (Fig. 6B). Morphological observation after $4 \mathrm{~h}$ of co-culture of the THP-1 cells and PMA-NETs, in which CD14 expression was induced by VD3 stimulation, showed that THP-1 cells phagocytosed NETs (Fig. 6C).

\section{Discussion}

It has been reported that when neutrophils and monocytes phagocytose infectious bacteria in blood vessels, CD14 expressed on their cell surface is taken up by the cells, degraded by neutrophil elastase, and released as presepsin into the blood ${ }^{[14]}$. However, there are reports of high presepsin levels in patients with SLE without infection, and the cause of this has not been clarified ${ }^{[18,19]}$. We focused on NETs, which are known to increase in the blood in sepsis and in SLE patients, to elucidate the production mechanism of increased presepsin even in SLE patients who do not have an infection. Hakkim et al. reported that the number of NETs in the blood was higher in patients with SLE due to less degradation of NETs than in healthy subjects ${ }^{[20]}$. In addition to the conventional mechanism of presepsin production, we hypothesize a novel mechanism of presepsin production in which monocytes/macrophages produce presepsin by phagocytosing dead NETs.

NETs were induced in a concentration-dependent manner in neutrophils isolated from the peripheral blood of healthy subjects by both DH5a and PMA. However, in the case of NET induction, the NET ratio did not increase up to a DH5a culture OD of 0.1 but increased at an OD of 1.0, suggesting that a certain amount of infection is necessary for NET induction. In septic patients, when the amount of infectious bacteria in the blood is low, the bacteria are eliminated only by the phagocytic activity of neutrophils and monocytes; however, when the amount of infectious bacteria is high, the NET defense mechanism is activated in addition to phagocytic activity ${ }^{[35]}$. The induction of NETs by DH5a at an OD of 1.0 is thought to replicate the appearance of NETs in sepsis in vitro.

NETs are formed in PMA-NETs by activating neutrophil protein kinase $\mathrm{C}$ (PKC) to activate NADPH oxidase [36]. The NET ratio increased in a concentration-dependent manner up to $100 \mathrm{nM}$ PMA treatment but did not increase significantly above $100 \mathrm{nM}$ PMA treatment. These results suggest that $100 \mathrm{nM}$ is an upper 
limit of PMA induced NADPH oxidase activity against neutrophils. NETs include suicidal NETs, which are ROS production-dependent, and vital NETs, which are non-ROS production-dependent. It has been reported that suicidal NETs release citrullinated histones into the extracellular space and die after the sterilization of bacteria ${ }^{[35,37]}$. In our experiments, we did not find any NETs with a morphology inferring that vital NETs were formed in both DH5a- and PMA-stimulation; instead, we found NETs with either NETtype I, in which the cytoplasm collapsed and trapped bacteria, or NET-type II, in which DNA was released from the cell and trapped bacteria. A larger number of DH5a-NETs showed NET-type II morphology than PMA-NETs. This may be because of the active induction and activation of NETs by neutrophils to capture more bacteria. Comparison of the expression of CD14, MPO, and NE on the surface of neutrophils by flow cytometry showed that compared with unstimulated neutrophils (untreated), neutrophils stimulated by DH5a were activated, and the expression of CD14, MPO, and NE was increased. Masuda et al have described the fractions with high forward scatter (FS) and side scatter (SS) that appeared after stimulation with $\mathrm{DH} 5 \mathrm{a}$ as the NET area ${ }^{[38,39]}$. The fact that in our experiments the ratio of neutrophils in the NET area increased during NET induction by DH5a or PMA suggests that NET formation was induced. In the NET area, CD14, MPO, and NE expressions were higher than that in DH5a-activated neutrophils, suggesting that cells in the NET area are highly capable of defending themselves by increasing the expressions CD14, which serves as a sensor for recognizing bacteria, and MPO and NE, which sterilize the bacteria.

MPO released from cells by NETs causes MPO cytoplasmic antibody (MPO-ANCA)-associated vasculitis, leading to a vicious inflammation cycle ${ }^{[40-43]}$. Therefore, presepsin, which can be measured easily and rapidly, could be used as a biomarker for these diseases. In studies on septic patients and mouse models, it has been reported that CD14 expression increases in an MYD88-dependent manner ${ }^{[13]}$. In our experiments, CD14 expression in neutrophils increased after stimulation with DH5a. Interestingly, flow cytometry and immunofluorescence imaging revealed that CD14 expression was higher in the NET areas. Braian et al. reported that macrophages phagocytosed Mycobacterium tuberculosis-induced NETs and monocytes/macrophages cleared NETs and produced presepsin in vivo ${ }^{[44]}$. Similarly, our immunofluorescence imaging using a presepsin antibody showed that monocytes phagocytosed NETs showing high CD14 levels in both DH5a- and PMA-stimulated NETs, demonstrating the production of presepsin in monocytes. However, compared to the isolated neutrophils, no significant change in presepsin levels was observed after induction of NETs by DH5a or PMA, suggesting that NETs do not produce presepsin; rather, monocytes/macrophages produce presepsin by phagocytosis of NETs. In particular, PMA-NETs only induced NETs in the absence of bacteria, which were phagocytosed by the monocytes, resulting in an increase in presepsin levels. These results indicate that monocytes phagocytose NETs that express high CD14 levels and produce presepsin in monocytes. In addition, when the NET number was increased in a PMA concentration-dependent manner and monocytes were phagocytosed, the presepsin level also increased as the NET ratio increased, demonstrating that the number of NETs had a significant effect on presepsin production. The induction of PMA-NETs was inhibited by DPI, an inhibitor of NADPH oxidase, and the presepsin levels decreased when the cells were co-cultured with monocytes. This result demonstrated that monocytes phagocytose NETs and thus 
produce presepsin and the number of NETs phagocytosed directly reflects the amount of presepsin produced under the same conditions in monocytes. Similar results were obtained for DH5a-NETs.

To prove that NETs were phagocytosed by monocytes in co-culture with NETs, we performed a phagocytosis inhibition test using cytochalasin $D$, which inhibits the actin polymerization of phagocytes. Presepsin production was inhibited when NETs were co-cultured with monocytes. This may be because monocytes cannot phagocytose NETs. In addition, when sivelestat, an inhibitor of neutrophil elastase, was added before co-culturing NETs with monocytes, presepsin production was suppressed. These results demonstrated that neutrophil elastase intracellularly degrades CD14 expressed by NETs after monocytes phagocytose NETs and presepsin is produced by monocytes to phagocytose NETs.

We hypothesized that macrophages in tissues also phagocytose NETs and produce presepsin, as the stagnation time of monocytes in peripheral blood is several hours in vivo. Presepsin levels decreased after inhibition of PMA-NETs using DPI in PMA-NET co-culture with macrophage-induced cell lines, suggesting that macrophage-induced cell lines produce presepsin via phagocytosis of NETs. In particular, differentiated machrophage THP-1 cells actively phagocytosed PMA-NETs and showed increased presepsin levels relative to those in U937 cells. We concluded that monocytes and macrophage cell lines phagocytose NETs and produce presepsin.

Collectively, these results indicate that when the level of NETs increases in the blood owing to sepsis or SLE, many of them undergo cell death, and monocytes/macrophages phagocytose NETs that express high CD14 levels on their cell surface. Thereafter, CD14 is degraded by neutrophil elastase in monocyte/macrophage cells to produce presepsin. Furthermore, in cases of increased presepsin levels in hemophagocytic syndrome, we speculated that phagocytes phagocytose CD14-expressing blood cells, resulting in increased presepsin levels (Fig. 7). The new mechanism of presepsin production is of great clinical significance because it not only explains the increased presepsin levels in SLE but also has the potential to be used as an indicator of SLE treatment efficacy. Further evidence on the mechanisms of the crosstalk between monocyte/macrophage and NETs will aid in the identification of novel therapeutic strategies for SLE and hemophagocytic syndrome.

\section{Methods}

The content and execution of the current study were approved by The Ethical Committee of the Kagawa Prefectural University of Health Sciences, Japan (No.327). All methods were carried out in accordance with the guidelines and regulations of The Ethical Committee of the Kagawa Prefectural University of Health Sciences. Written informed consent was obtained from the participants before the study.

\section{Healthy donor samples and cell lines culture.}

The study was approved by the ethics committee of Kagawa Prefectural University of Health Sciences, and consent was obtained from the participating students and teaching staff $(n=7)$. U937 cells (American Type Culture Collection: CRL-1593.2) and THP-1 cells (American Type Culture Collection: TIB- 
202) were grown in RPMI1640 medium (Cat, No. R8758; Sigma-Aldrich, St. Louis, MO, USA) containing $10 \%(\mathrm{v} / \mathrm{v})$ heat-inactivated fetal bovine serum (FBS) in a humidified atmosphere with $5 \% \mathrm{CO}_{2}$ at $37^{\circ} \mathrm{C}$.

\section{Antibodies, proteins, and chemicals.}

The details of reagents used in this study and their sources are as follows: diphenyleneiodonium chloride (DPI; Cat, No.81050; Cayman Chemical Company, Ann Arbor, MI, USA), cytochalasin D (Cat, No.11330; Cayman Chemical Company), sivelestat (Cat, No.17779; Cayman Chemical Company), phorbol 12myristate 13-acetate (PMA; Cat, No.P8139; Sigma-Aldrich), 1a, 25-dihydroxyvitamin D3 (VD3; Cat, No.71820; Cayman Chemical Company), May-Grünwald's stain solution (Cat, No.15053; Mutokagaku, Tokyo, Japan), Gimsa's stain solution (Cat, No.15002; Mutokagaku), 1/15M phosphate buffer solution (pH 6.4) (Cat, No.15612; Mutokagaku), DAB Stain Kit ( Cat, No.15712; Mutokagaku), anti-human myeloperoxidase mouse monoclonal antibody (Cat, No.sc-52707; Santa Cruz Biotechnology, Dallas, TX, USA), anti-human neutrophil elastase mouse monoclonal antibody (Cat, No.sc-55549; Santa Cruz Biotechnology), anti-human citrullinated histone H3 rabbit polyclonal antibody (Cat, No.ab5103; Abcam, Cambridge, UK), anti-human presepsin mouse monoclonal antibody (F1106-13-3 antibody; Mochida Seiyaku, Tokyo, Japan), anti-human CD14 mouse monoclonal antibody (Cat, No.14-0149-82; Invitrogen, Carlsbad, CA, USA), FITC-conjugated anti-human CD14 mouse monoclonal antibody (Cat, No.6603511; Beckman Coulter, Brea, CA, USA), Alexa Flour 405-conjugated goat anti-rabbit IgG (Cat, No.ab175652, Invitrogen), Rhodamine (TRITC) -conjugated goat anti-mouse IgG (Cat, No.SA00007-1; Cosmobio, Tokyo, Japan), and E. Coli DH5a Competent Cells (Cat, No.9057; Takara Bio Shiga, Japan).

\section{Isolation of neutrophils.}

Neutrophils were isolated at room temperature from the EDTA-anticoagulated peripheral blood of healthy volunteers by density gradient centrifugation using Polymorphprep (Cat, No.1114683; Axis-Shield, Dundee, Scotland). After centrifugation for $30 \mathrm{~min}$ at $500 \times \mathrm{g}$, the lower cellular fraction containing neutrophils was collected, serum-free RPMI 1640 medium was added, and the neutrophils were washed by centrifugation for $10 \mathrm{~min}$ at $400 \times \mathrm{g}$.

\section{Isolation of monocytes from PBMCs.}

After density gradient centrifugation using Polymorphprep, the upper cell fraction containing peripheral blood mononuclear cells (PBMCs) was collected, serum-free RPMI 1640 medium was added, and the cells were washed twice by centrifugation for $10 \mathrm{~min}$ at $100 \times g$ to remove platelets. Thereafter, monocytes were isolated from purified PBMCs using the EasySep Human Monocyte Isolation Kit (Cat. No. 19359; Stemcell Technologies, Vancouver, BC, Canada) according to the manufacturer's instructions.

\section{Immobilization and optical density of E. coli DH5a competent cells.}

Escherichia coli DH5a competent cells (DH5a) were used after formaldehyde fixation. To standardize the amount of immobilized DH5a, the optical density (OD) was measured and adjusted. The number of DH5a 
bacteria at $\mathrm{OD} 1.0$ was equivalent to $3.0 \times 10^{5} / \mu \mathrm{L}$, as determined from the colony assay. For the induction of NET formation, $100 \mu \mathrm{L}$ of the bacterial solution was added to $900 \mu \mathrm{L}$ of the neutrophil suspension.

\section{Induction of NETs and measurement of the NET ratio.}

Purified neutrophils $\left(1.8 \times 10^{6}\right.$ cells/well) were seeded in 24-well plates (Cat. No. SIAL0526; SigmaAldrich) and stimulated using an OD-adjusted DH5a bacterial solution (DH5a-NETs) or 50 nM PMA (PMANETs). After stimulation with OD $1.0 \mathrm{DH} 5 \mathrm{a}$ for $4 \mathrm{~h}$ at $37^{\circ} \mathrm{C}$ or $50 \mathrm{nM} \mathrm{PMA}$ for $2 \mathrm{~h}$ at $37^{\circ} \mathrm{C}$, SYTOX Green was added to $100 \mu \mathrm{L}$ of the cell suspension and incubated for $20 \mathrm{~min}$ at room temperature, and the cells were analyzed using a flow cytometer (Cell Lab Quanta SC; Beckman Coulter) without washing. The NET area was defined as the fraction of cells with high side and forward scatter and positive extracellular SYTOX Green nuclear staining was compared with untreated neutrophils. The SYTOX Green positivity rate was evaluated as the NET ratio ${ }^{[38,45]}$.

\section{Cellular morphology in NETs.}

Purified neutrophils were seeded in 24-well plates with submerged glass discs at the bottom, and NETs were induced using DH5a and PMA. The induced NETs were May-Giemsa-stained, myeloperoxidasestained, and observed under a microscope (OLYMPUS Corporation, BX50, Tokyo Japan) at 1,000x magnification. Staining was performed according to the manufacturer's instructions.

\section{Measurement of CD14, MPO, and NE expression in NETs.}

The expression levels of CD14, myeloperoxidase (MPO), and neutrophil elastase (NE) in neutrophils (untreated) and NET areas after $4 \mathrm{~h}$ of $\mathrm{DH} 5 \mathrm{a}$ addition were compared using mean fluorescence intensity.

\section{Evaluation of Presepsin levels by co-culturing with monocytes after NET induction.}

Purified monocytes (5.0 $\times 10^{5}$ cells per well) were seeded in 24-well plates, and the same number of purified neutrophils (control) and NETs from the peripheral blood of the same subject were added after incubation for $3 \mathrm{~h}$ at $37^{\circ} \mathrm{C}$, and presepsin in the supernatant was measured using PATHFAST (LSI Medience Corporation, Tokyo, Japan).

\section{Immunofluorescence imaging of NETs.}

Purified neutrophils were seeded in 24-well plates with submerged glass discs at the bottom, and NETs were induced using DH5a and PMA. After stimulation, the medium was removed, and the remaining cells were washed with phosphate-buffered saline (PBS). Cells on the glass discs were fixed with $4 \%$ paraformaldehyde for 10 min at room temperature. After washing with PBS, cells were incubated in PBS containing $5 \%$ rat serum for 60 min to block non-specific antibody binding. The samples were then incubated for 60 min with the following primary antibodies: anti-human citrullinated histone $\mathrm{H} 3$ (Cit-H3) rabbit polyclonal antibody (dilution 1:1,000), anti-human MPO mouse monoclonal antibody (dilution 1:2,000) or anti-human NE mouse monoclonal antibody (dilution 1:2,000) or anti-human CD14 mouse 
monoclonal antibody (dilution 1:500). After washing in PBS, each primary antibody binding was visualized using secondary antibodies coupled to Alexa Fluor 405-conjugated goat anti-rabbit lgG (dilution 1:1,000) and rhodamine (TRITC)-conjugated goat anti-mouse IgG (dilution 1:2,000), and the samples were stained for DNA (Sytox Green; dilution 1:2,000). After incubation for $60 \mathrm{~min}$, samples were washed with PBS and embedded in $80 \%$ glycerol. All the procedures were performed at room temperature. Images were obtained using a confocal microscope (FLUOVIEW FV10i; Olympus Corporation, Tokyo, Japan).

\section{Immunofluorescence imaging of monocytes that had phagocytosed NETs.}

Purified monocytes were seeded in 24-well plates with glass discs submerged at the bottom and cocultured with monocytes after induction of NET formation. After incubation for $3 \mathrm{~h}$ at $37^{\circ} \mathrm{C}$, the medium was removed, and the remaining cells were washed with PBS. Cells on the glass discs were fixed with $4 \%$ paraformaldehyde. After washing with PBS, samples were incubated in PBS containing $5 \%$ rat serum for $60 \mathrm{~min}$. Thereafter, the samples were incubated for $60 \mathrm{~min}$ with the following primary antibodies: antihuman cit-H3 rabbit polyclonal antibody (dilution, 1:1,000) and anti-human presepsin mouse monoclonal antibody (dilution, 1:5,000). After washing in PBS, each primary antibody binding was visualized using secondary antibodies coupled to Alexa Fluor 405-conjugated goat anti-rabbit IgG (dilution 1:1,000) and rhodamine (TRITC)-conjugated goat anti-mouse IgG (dilution 1:2,000). Subsequently, the samples were stained for DNA (Sytox Green; dilution 1:2,000) or CD14 using FITC-conjugated anti-human CD14 mouse monoclonal antibody. After incubation for 60 min, samples were washed with PBS and embedded in $80 \%$ glycerol. All the procedures were performed at room temperature. Images were acquired using a confocal microscope (FLUOVIEW FV10i).

\section{NET ratio and presepsin evaluation after inhibitor treatment.}

NETs were treated with DPI, which inhibits NADPH oxidase activity. Before DH5a or PMA stimulation, purified neutrophils $\left(1.8 \times 10^{6}\right.$ cells/well) were exposed to $10 \mu \mathrm{M} \mathrm{DPI}$ for $30 \mathrm{~min}$ at $37^{\circ} \mathrm{C}$. After NET inhibition, the NET ratio was analyzed by flow cytometry, and presepsin levels were measured after monocyte phagocytosis. Cytochalasin D was used as a phagocytosis inhibitor, and sivelestat was used as an neutrophil elastase inhibitor. Before phagocytosis of NETs by monocytes, purified monocytes $(5.0 \times$ $10^{5}$ cells/well) were exposed to $50 \mu \mathrm{M}$ cytochalasin $\mathrm{D}$ or $50 \mu \mathrm{M}$ sivelestat for $30 \mathrm{~min}$ at $37^{\circ} \mathrm{C}$. After coculture with NETs, the presepsin levels were measured.

\section{NET phagocytosis by macrophages increases presepsin levels.}

To differentiate U937 and THP-1 cells into active macrophage-like cells, the cells were resuspended in a culture medium containing $100 \mathrm{nM}$ VD3 to a density of $2.0 \times 10^{5}$ cells $/ \mathrm{mL}$ and incubated for $48 \mathrm{~h}$ at $37^{\circ} \mathrm{C}$. After differentiation, the cells were co-cultured with PMA-NETs derived from neutrophils obtained from healthy volunteers for $3 \mathrm{~h}$, and presepsin in the supernatant was measured.

\section{Statistical analyses.}


All statistical analyses were performed using SPSS version 24.0 (SPSS Inc, Chicago, IL, USA). The data are presented as the mean \pm standard deviation (SD), and Student's $t$-test was used for comparisons between two groups. A $p$-value of less than 0.05 was considered statistically significant. In the graphically represented data, ${ }^{*}$, and $* \star$ denote $p$ values of less than $0.05,0.01$, respectively.

\section{Declarations}

\section{Acknowledgments}

We are grateful to Mr. Takefumi Sano and Mr. Kamon Shirakawa for their technical support.

\section{Author contributions}

Study conception and design: A.I. and A.K. Acquisition of data: K.K. and K.S. Interpretation of data: M.M.

\section{Data availability statement}

On reasonable request to the corresponding author, data supporting the findings of this study will be available after approval from the Ethical Committee of the Kagawa Prefectural University of Health Sciences.

\section{Competing interests}

The authors declare no competing interests.

\section{References}

1. Dellinger, R. P. et al. Surviving sepsis campaign: international guidelines for management of severe sepsis and septic shock:2012. Crit. Care Med. 41, 580-637 (2013). 10.1097/CCM.0b013e31827e83af, Pubmed:23353941.

2. Cecconi, M., Evans, L., Levy, M. \& Rhodes, A. Sepsis and septic shock. Lancet 392, 75-87 (2018). 10.1016/S0140-6736(18)30696-2, Pubmed:29937192.

3. Husabø, G. et al. Early diagnosis of sepsis in emergency departments, time to treatment, and association with mortality: an observational study. PLOS ONE 15, e0227652 (2020). 10.1371/journal.pone.0227652, Pubmed:31968009.

4. Hilarius, K. W. E., Skippen, P. W. \& Kissoon, N. Early recognition and emergency treatment of sepsis and septic shock in children. Pediatr. Emerg. Care 36, 101-106 (2020). 10.1097/PEC.0000000000002043, Pubmed:32011568.

5. Landmann, R., Müller, B. \& Zimmerli, W. CD14, new aspects of ligand and signal diversity. Microbes Infect. 2, 295-304 (2000). 10.1016/s1286-4579(00)00298-7, Pubmed:10758406.

6. Ziegler-Heitbrock, H. W. \& Ulevitch, R. J. CD14: cell surface receptor and differentiation marker. Immunol. Today 14, 121-125 (1993). 10.1016/0167-5699(93)90212-4, Pubmed:7682078. 
7. Antal-Szalmas, P. A., Strijp, J. A., Weersink, A. J., Verhoef, J. \& Van Kessel, K. P. Quantitation of surface CD14 on human monocytes and neutrophils. J. Leukoc. Biol. 61, 721-728 (1997). 10.1002/jlb.61.6.721, Pubmed:9201263.

8. Labeta, M. O., Landmann, R., Obrecht, J. P. \& Obrist, R. Human B cells express membrane-bound and soluble forms of the CD14 myeloid antigen. Mol. Immunol. 28, 115-122 (1991). 10.1016/01615890(91)90094-z, Pubmed:1707133.

9. Haziot, A., Tsuberi, B. Z. \& Goyert, S. M. Neutrophil CD14: biochemical properties and role in the secretion of tumor necrosis factor-alpha in response to lipopolysaccharide. J. Immunol. 150, 55565565 (1993). Pubmed:7685797.

10. Chaby, R., Metezeau, P. \& Girard, R. Binding of a rhodamine-labeled lipopolysaccharide to lipopolysaccharide-responder and nonresponder lymphocytes. Cell. Immunol. 85, 531-541 (1984). 10.1016/0008-8749(84)90265-x, Pubmed:6201292.

11. Mussap, M., Noto, A., Fravega, M. \& Fanos, V. Soluble CD14 subtype presepsin (sCD14-ST) and lipopolysaccharide binding protein (LBP) in neonatal sepsis: new clinical and analytical perspectives for two old biomarkers. J. Matern. Fetal Neonatal Med. 24 Supplement 2, 12-14 (2011). 10.3109/14767058.2011.601923, Pubmed:21740312.

12. Nakazawa, D. et al. Abnormal conformation and impaired degradation of propylthiouracil-induced neutrophil extracellular traps: implications of disordered neutrophil extracellular traps in a rat model of myeloperoxidase antineutrophil cytoplasmic antibody-associated vasculitis. Arthritis Rheum. 64, 3779-3787 (2012). 10.1002/art.34619, Pubmed:22777766.

13. Chen, Z. et al. Sepsis upregulates CD14 expression in a MyD88-dependent and Trif-independent pathway. Shock 49, 82-89 (2018). 10.1097/SHK.0000000000000913, Pubmed:28562479.

14. Arai, Y. et al. Phagocytosis by human monocytes is required for the secretion of presepsin. J. Infect. Chemother. 21, 564-569 (2015). 10.1016/j.jiac.2015.04.011, Pubmed:26026662.

15. Shirakawa, K., Naitou, K., Hirose, J., Takahashi, T. \& Furusako, S. Presepsin (sCD14-ST): development and evaluation of one-step ELISA with a new standard that is similar to the form of presepsin in septic patients. Clin. Chem. Lab. Med. 49, 937-939 (2011). 10.1515/CCLM.2011.145, Pubmed:21345045.

16. Yaegashi, Y. et al. Evaluation of a newly identified soluble CD14 subtype as a marker for sepsis. J. Infect. Chemother. 11, 234-238 (2005). 10.1007/s10156-005-0400-4, Pubmed:16258819.

17. Endo, S. et al. Usefulness of presepsin in the diagnosis of sepsis in a multicenter prospective study. J. Infect. Chemother. 18, 891-897 (2012). 10.1007/s10156-012-0435-2, Pubmed:22692596.

18. Lood, C. et al. Neutrophil extracellular traps enriched in oxidized mitochondrial DNA are interferogenic and contribute to lupus-like disease. Nat. Med. 22, 146-153 (2016). 10.1038/nm.4027, Pubmed:26779811.

19. Tanimura, S. et al. Clinical significance of plasma presepsin levels in patients with systemic lupus erythematosus. Mod. Rheumatol. 28, 865-871 (2018). 10.1080/14397595.2017.1408755, Pubmed:29239264. 
20. Hakkim, A. et al. Impairment of neutrophil extracellular trap degradation is associated with lupus nephritis. Proc. Natl Acad. Sci. U. S. A. 107, 9813-9818 (2010). 10.1073/pnas.0909927107, Pubmed:20439745.

21. Brinkmann, V. et al. Neutrophil extracellular traps kill bacteria. Science 303, 1532-1535 (2004). 10.1126/science.1092385, Pubmed:15001782.

22. Kaplan, M. J. \& Radic, M. Neutrophil extracellular traps: double-edged swords of innate immunity. J. Immunol. 189, 2689-2695 (2012). 10.4049/jimmunol.1201719, Pubmed:22956760.

23. Ravindran, M., Khan, M. A. \& Palaniyar, N. Neutrophil extracellular trap formation: physiology, pathology, and pharmacology. Biomolecules 9, 365 (2019). 10.3390/biom9080365, Pubmed:31416173.

24. Nakashima, K., Hagiwara, T. \& Yamada, M. Nuclear localization of peptidylarginine deiminase $V$ and histone deimination in granulocytes. J. Biol. Chem. 277, 49562-49568 (2002). 10.1074/jbc.M208795200, Pubmed:12393868.

25. Vorobjeva, N. V. \& Chernyak, B. V. NETosis: molecular mechanisms, role in physiology and pathology. Biochemistry (Mosc) 85, 1178-1190 (2020). 10.1134/S0006297920100065, Pubmed:33202203.

26. Borregaard, N. Neutrophils, from marrow to microbes. Immunity 33, 657-670 (2010). 10.1016/j.immuni.2010.11.011, Pubmed:21094463.

27. Denning, N. L., Aziz, M., Gurien, S. D. \& Wang, P. DAMPs and NETs in sepsis. Front. Immunol. 10, 2536 (2019). 10.3389/fimmu.2019.02536, Pubmed:31736963.

28. Schauer, C. et al. Aggregated neutrophil extracellular traps limit inflammation by degrading cytokines and chemokines. Nat. Med. 20, 511-517 (2014). 10.1038/nm.3547, Pubmed:24784231.

29. Remijsen, Q. et al. Neutrophil extracellular trap cell death requires both autophagy and superoxide generation. Cell Res. 21, 290-304 (2011). 10.1038/cr.2010.150, Pubmed:21060338.

30. Xu, J. et al. Extracellular histones are major mediators of death in sepsis. Nat. Med. 15, 1318-1321 (2009). 10.1038/nm.2053, Pubmed:19855397.

31. Ammollo, C. T., Semeraro, F., Xu, J., Esmon, N. L. \& Esmon, C. T. Extracellular histones increase plasma thrombin generation by impairing thrombomodulin-dependent protein $\mathrm{C}$ activation. $\mathrm{J}$. Thromb. Haemost. 9, 1795-1803 (2011). 10.1111/j.1538-7836.2011.04422.x, Pubmed:21711444.

32. Brill, A. et al. Neutrophil extracellular traps promote deep vein thrombosis in mice. J. Thromb. Haemost. 10, 136-144 (2012). 10.1111/j.1538-7836.2011.04544.x, Pubmed:22044575.

33. Semeraro, F. et al. Extracellular histones promote thrombin generation through platelet-dependent mechanisms: involvement of platelet TLR2 and TLR4. Blood 118, 1952-1961 (2011). 10.1182/blood2011-03-343061, Pubmed:21673343.

34. Leffler, J. et al. Neutrophil extracellular traps that are not degraded in systemic lupus erythematosus activate complement exacerbating the disease. J. Immunol. 188, 3522-3531 (2012). 10.4049/jimmunol.1102404, Pubmed:22345666. 
35. Manda-Handzlik, A. M. et al. Flow cytometric quantification of neutrophil extracellular traps: limitations of the methodological approach. Am. J. Hematol. 91, E9-E10 (2016). 10.1002/ajh.24257, Pubmed:26616047.

36. Masuda, S. et al. Measurement of NET formation in vitro and in vivo by flow cytometry. Cytometry A 91, 822-829 (2017). 10.1002/cyto.a.23169, Pubmed:28715618.

37. Yipp, B. G. et al. Infection-induced NETosis is a dynamic process involving neutrophil multitasking in vivo. Nat. Med. 18, 1386-1393 (2012). 10.1038/nm.2847, Pubmed:22922410.

38. Fuchs, T. A. et al. Novel cell death program leads to neutrophil extracellular traps. J. Cell Biol. 176, 231-241 (2007). 10.1083/jcb.200606027, Pubmed:17210947.

39. Yousefi, S., Mihalache, C., Kozlowski, E., Schmid, I. \& Simon, H. U. Viable neutrophils release mitochondrial DNA to form neutrophil extracellular traps. Cell Death Differ. 16, 1438-1444 (2009). 10.1038/cdd.2009.96, Pubmed:19609275.

40. Masuda, S. et al. NETosis markers: quest for specific, objective, and quantitative markers. Clin. Chim. Acta 459, 89-93 (2016). 10.1016/j.cca.2016.05.029, Pubmed:27259468.

41. Kessenbrock, K. et al. Netting neutrophils in autoimmune small-vessel vasculitis. Nat. Med. 15, 623625 (2009). 10.1038/nm.1959, Pubmed:19448636.

42. Söderberg, D. \& Segelmark, M. Neutrophil extracellular traps in ANCA-associated vasculitis. Front. Immunol. 7, 256 (2016). 10.3389/fimmu.2016.00256, Pubmed:27446086.

43. Yoshida, M. et al. Myeloperoxidase anti-neutrophil cytoplasmic antibody affinity is associated with the formation of neutrophil extracellular traps in the kidney and vasculitis activity in myeloperoxidase anti-neutrophil cytoplasmic antibody-associated microscopic polyangiitis. Nephrology (Carlton) 21, 624-629 (2016). 10.1111/nep.12736, Pubmed:26833773.

44. O'Sullivan, K. M. \& Holdsworth, S. R. Neutrophil extracellular traps: A potential therapeutic target in MPO-ANCA associated vasculitis? Front. Immunol. 12, 635188 (2021). 10.3389/fimmu.2021.635188.

45. Braian, C., Hogea, V. \& Stendahl, O. Mycobacterium tuberculosis- induced neutrophil extracellular traps activate human macrophages. J. Innate Immun. 5, 591-602 (2013). 10.1159/000348676, Pubmed:23635526.

\section{Figures}


Fig.1
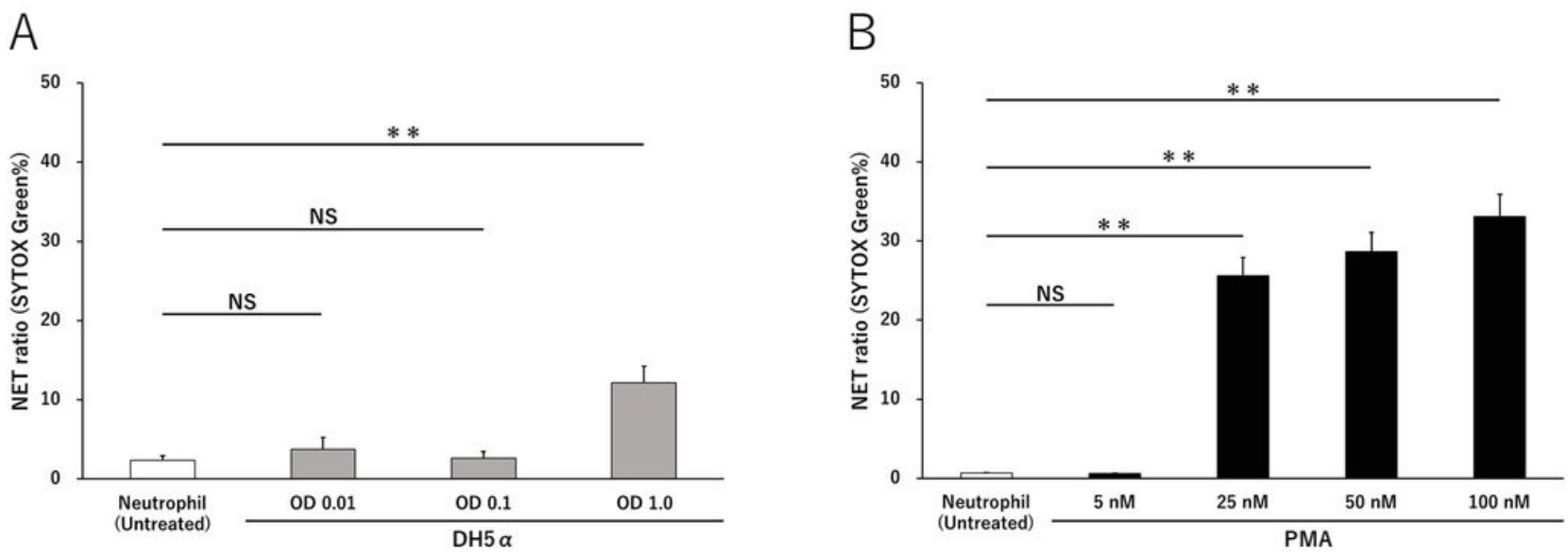

\section{Figure 1}

\section{NETs increase in a DH5a or PMA concentration-dependent manner.}

NETs were induced in a concentration-dependent manner by bacterial (DH5a) and PMA stimulation. (A) DH5a cells were incubated with neutrophils for $4 \mathrm{~h}$ under platelet-free conditions, and the NET ratio was measured. (B) The NET ratio in PMA stimulation was measured after $2 \mathrm{~h}$ of incubation with neutrophils under platelet-free conditions.

${ }^{* \star} p<0.001$, when compared with untreated neutrophils. Data represent the mean values \pm SD of at least three experiments. NS, not significant. 
Fig.2

A

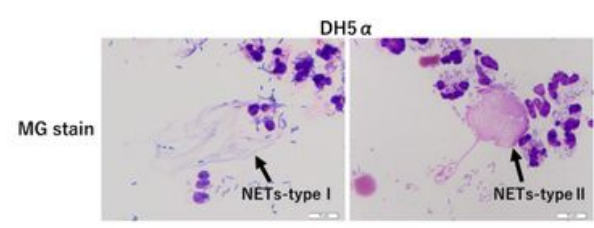

B

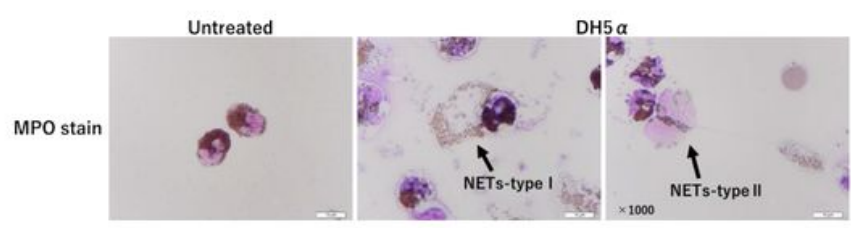

Fig.2

D

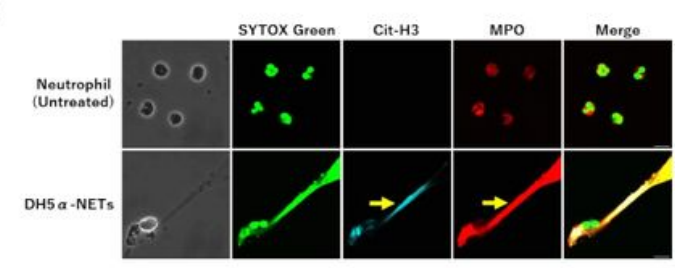

$\mathrm{E}$

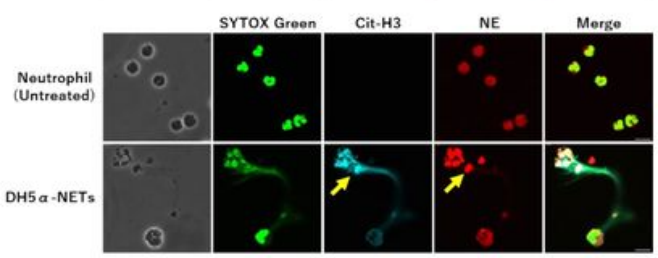

Fig.2

C
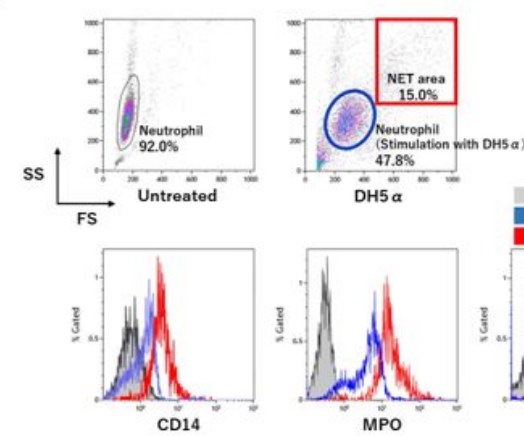

Neutrophil (Untreated)

NET area

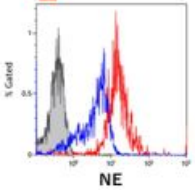

Fig.2

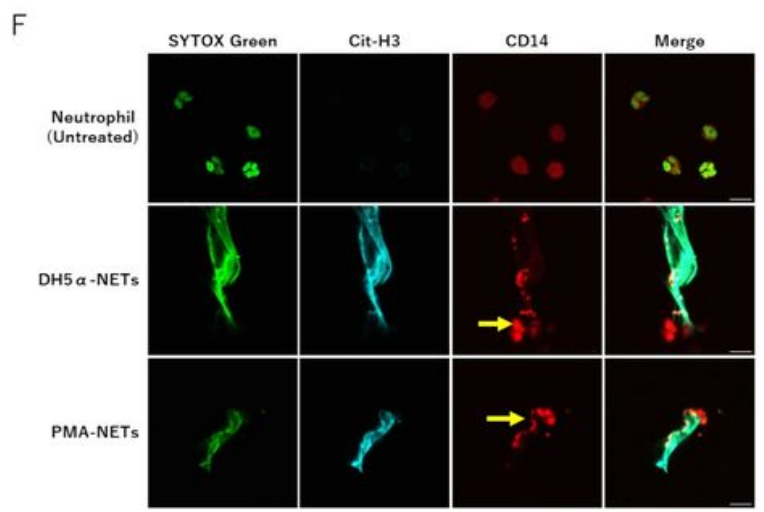

Figure 2

NETs with different cell morphology were observed in both DH5a and PMA-stimulated NETs.

(A, B) NET-type I, in which the nuclear structure is preserved, and the cytoplasm shows a disintegrated image, and NET-type II, in which DNA is released into the extracellular space. Scale bar: $10 \mu \mathrm{m}$. (C) The NET area highly expressed CD14, MPO, and NE than the neutrophils, as shown by flow cytometry. (D, E) Scale bar: $10 \mu \mathrm{m}$. Extracellular MPO and NE expression in DH5a-NETs by immunofluorescence imaging. (F) CD14 expression in DH5a-NETs and PMA-NETs. Scale bar: $10 \mu \mathrm{m}$. 
Fig.3

A

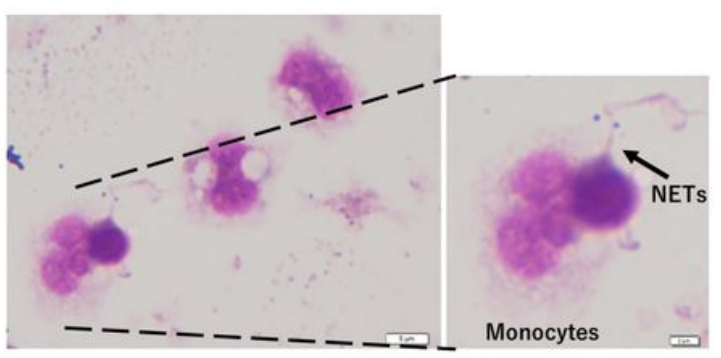

Fig.3

B

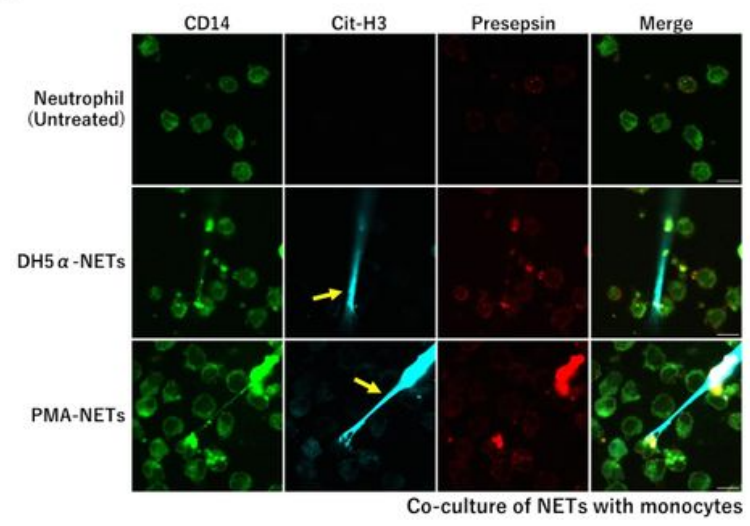

Fig.3

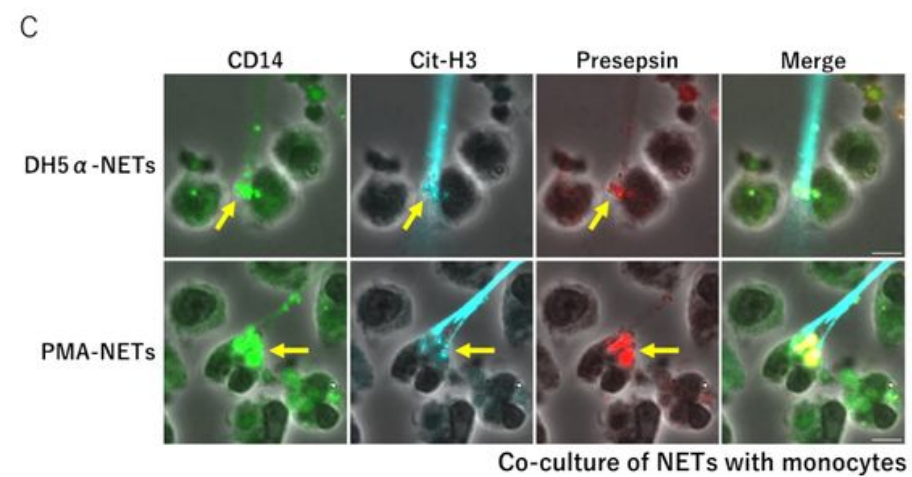

Fig.3
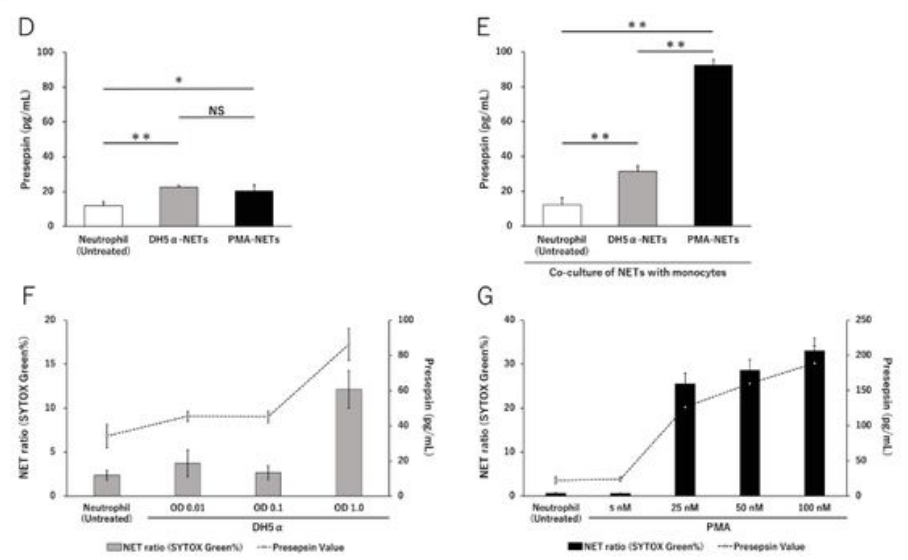

Figure 3

\section{Presepsin levels increase as the monocytes phagocytose NETs.}

(A) A May-Giemsa-stained image $3 \mathrm{~h}$ after PMA-NETs were induced and co-cultured with monocytes. $(1,000 \times$ magnification). The scale bar is $5 \mu \mathrm{m}$, and the scale bar in the magnified image is $2 \mu \mathrm{m}$. (B) Immunofluorescence imaging of co-culture with monocytes after induction of NETs. The arrow shows cells positive for citrullinated histones. Scale bar: $10 \mu \mathrm{m}$. (C) The arrow point indicates the area where monocytes are phagocytosing NETs. The scale bar in the magnified image is $5 \mu \mathrm{m}$. (D) Presepsin levels in DH5a (OD 1.0)-NET induction (NET ratio 14\%), presepsin levels in PMA (50 nM)-NETs induction (NET ratio 23\%). (E) Comparison of presepsin levels after phagocytosis by co-culturing with monocytes for $3 \mathrm{~h}$. $(F, G)$ Variation in NET ratio and presepsin values with DH5a and PMA concentrations are shown. * $p<$ 0.05 and ${ }^{* \star} p<0.001$, when compared with untreated neutrophils. Data represent the mean values \pm SD of at least three experiments. NS, not significant. 
Fig.4

A

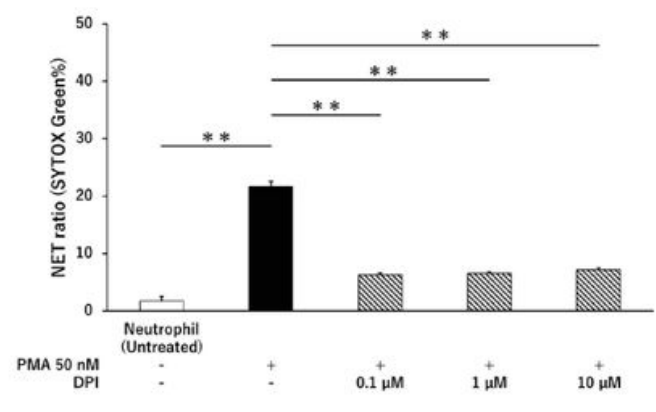

C

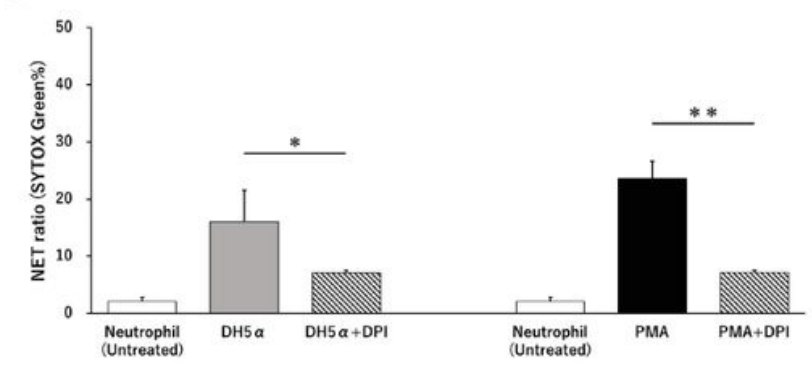

B

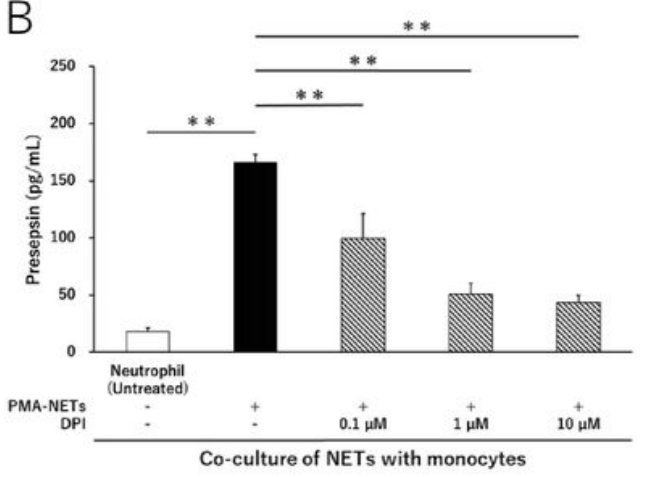

D

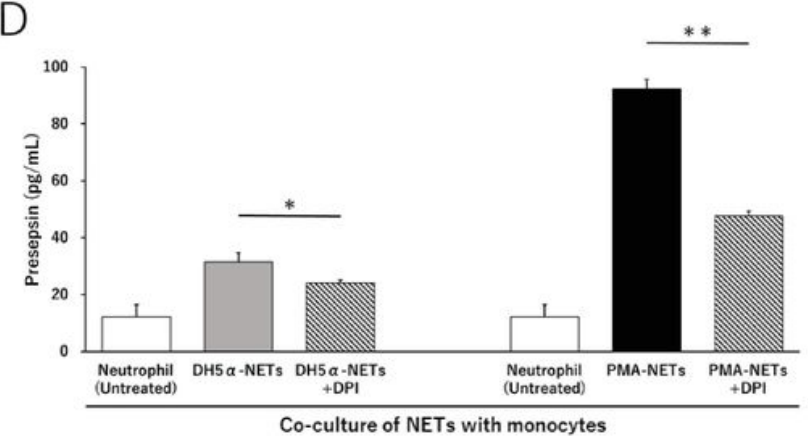

Figure 4

Presepsin levels decrease after NET suppression followed by co-culture with monocytes.

(A) After neutrophils were treated with DPI for 30 min, the NET ratio in PMA stimulation was measured.

(B) Comparison of presepsin levels after phagocytosis by co-culturing with monocytes for $3 \mathrm{~h}$. (C) NET ratio by $\mathrm{DH} 5 \mathrm{a}(\mathrm{OD} 1.0)$ and PMA (50 nM) stimulation after DPI $(10 \mu \mathrm{M})$ treatment. (D) After DH5a (OD 1.0)-NETs and PMA (50 nM)-NETs were suppressed by DPI $(10 \mu \mathrm{M})$, presepsin levels were compared when the cells were co-cultured with monocytes for $3 \mathrm{~h}$. ${ }^{\star} p<0.05$ and ${ }^{*} p<0.001$, compared with untreated neutrophils. Data represent the mean values \pm SD of at least three experiments. 


\section{Fig.5}

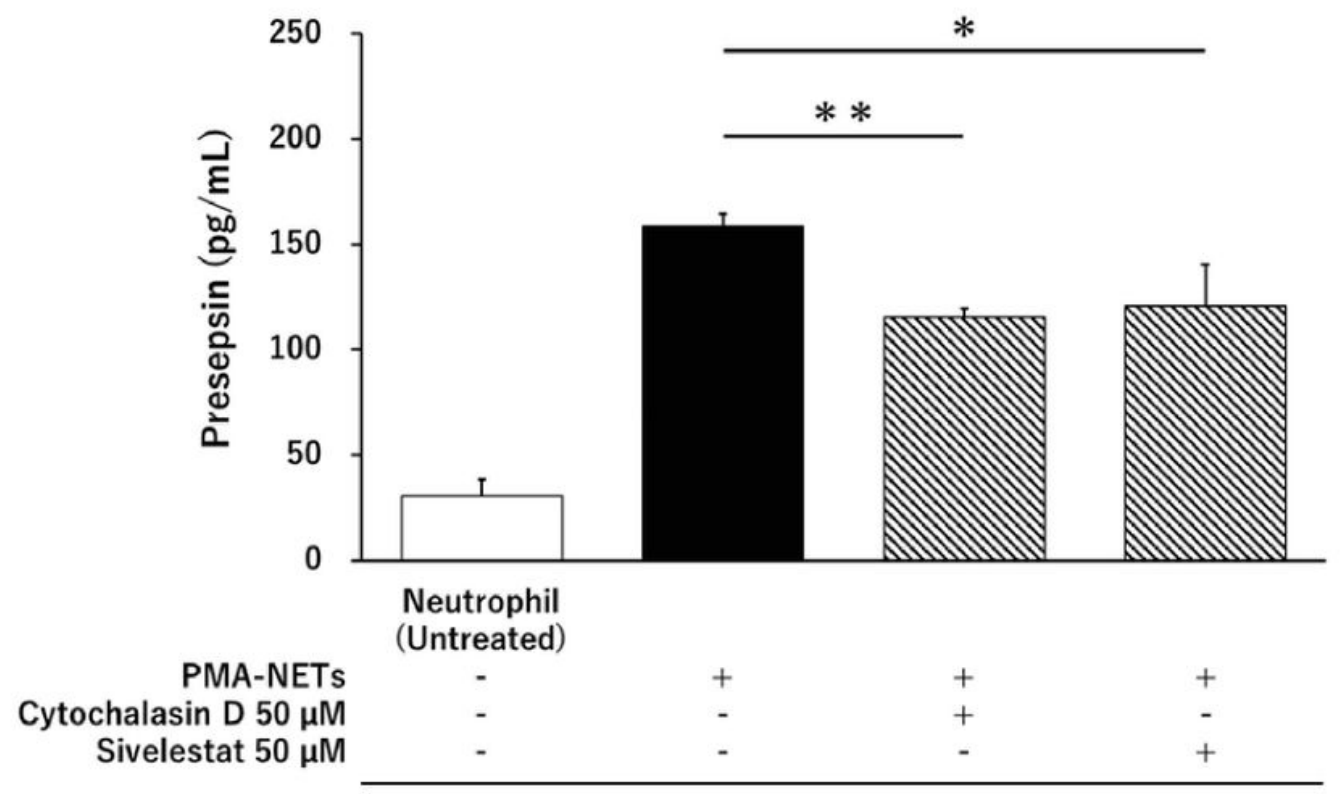

Co-culture of NETs with monocytes

\section{Figure 5}

Inhibiting the phagocytic activity of monocytes decreases presepsin levels.

Effect of phagocytosis and neutrophil elastase inhibitors on presepsin production by monocytes after induction of NETs with PMA (50 nM). ${ }^{*} p<0.05$ and ${ }^{* *} p<0.001$, compared with untreated neutrophils. Data represent the mean values \pm SD of at least three experiments. 
Fig.6

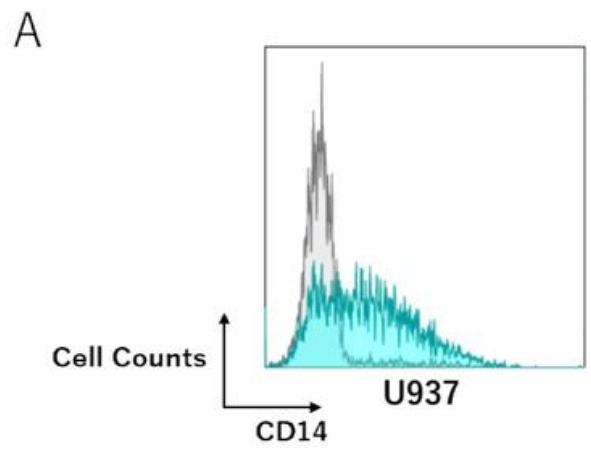

B

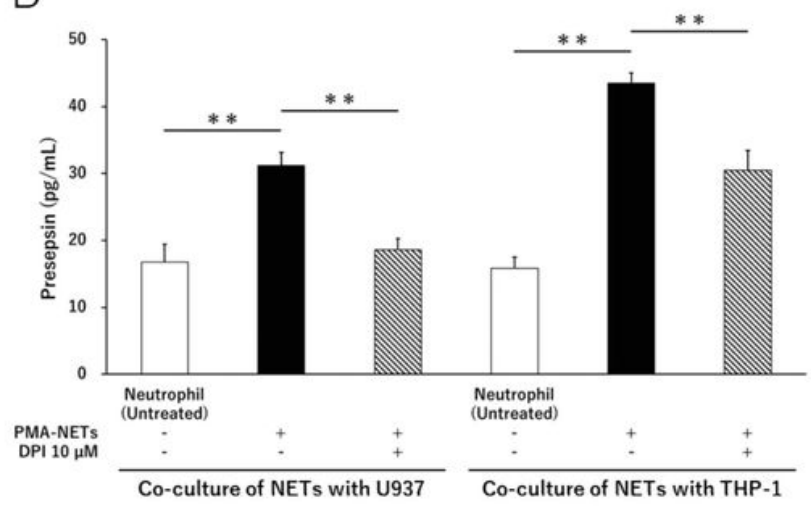

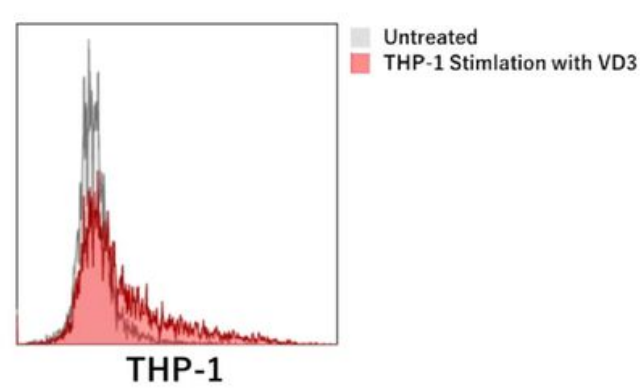

C

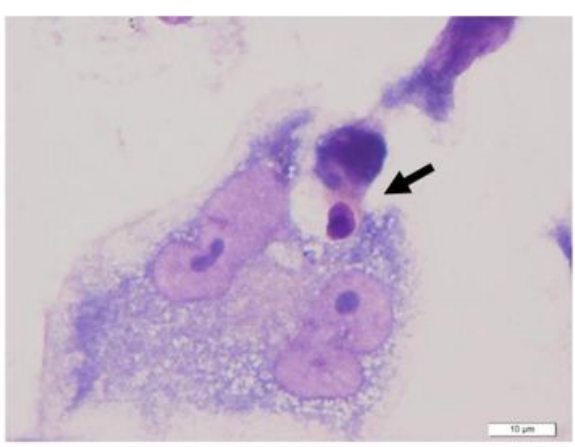

Figure 6

Presepsin levels increase as macrophages phagocytose NETs and decrease when NETs are suppressed.

(A) Differentiation into macrophages used VD3 to induce CD14 expression against U937 and THP-1 cell lines. (B) Comparison of presepsin levels when co-cultured with PMA-NETs and macrophages. (C) Phagocytosis of PMA-NETs by THP-1 after macrophage induction visualized May-Giemsa staining. Scale bar: $10 \mu \mathrm{m} .{ }^{*} p<0.001$, compared with untreated neutrophils. Data represent the mean values \pm SD of at least three experiments. 
Fig.7

\section{Monocyte/Mcrophage}

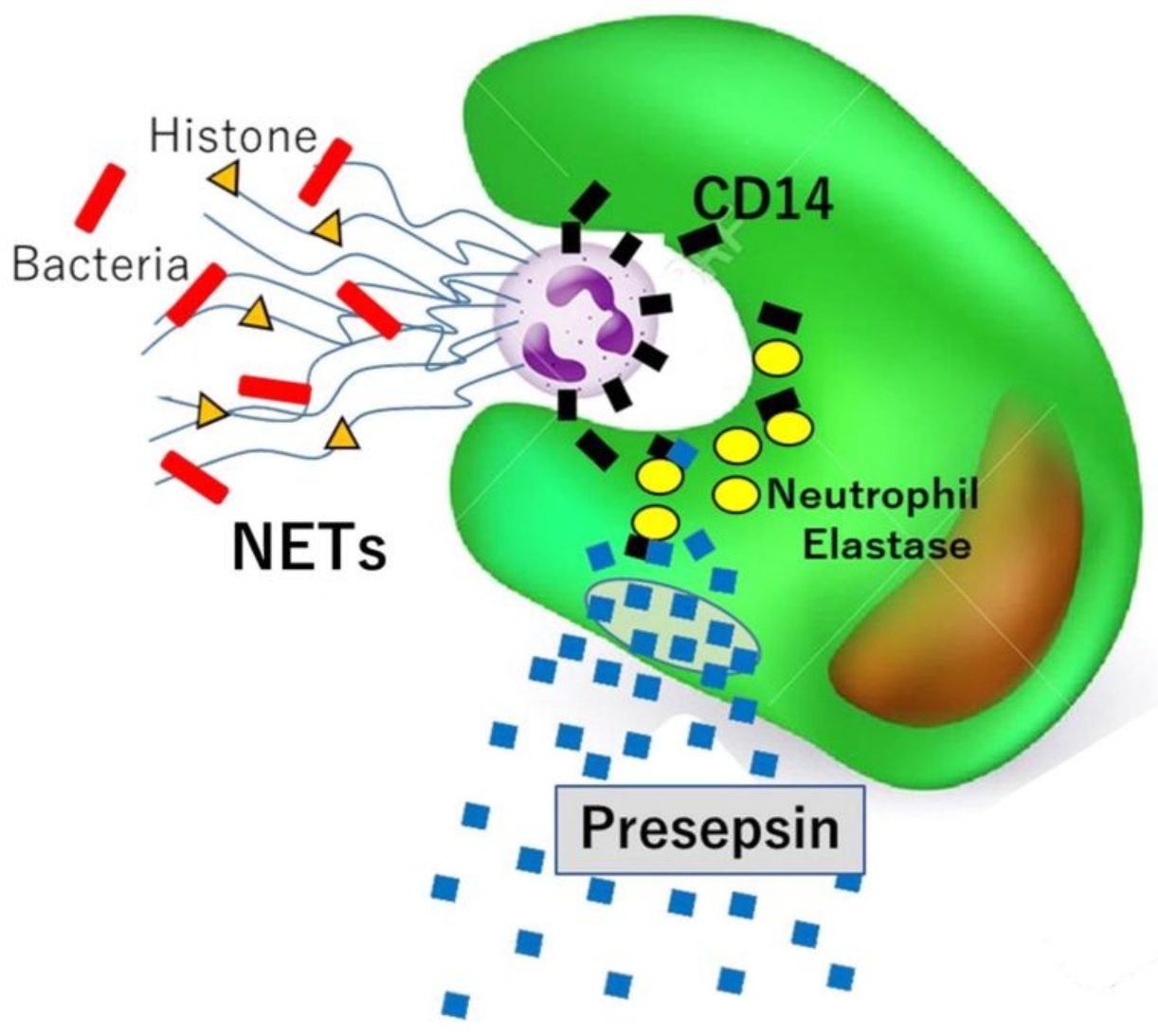

Figure 7

Schematic representation of the mechanism of presepsin production by monocytes/macrophages by NETphagocytosis.

Neutrophils invoke NETs in response to infectious bacteria in blood vessels, trapping bacteria and turning them into dead cells. Phagocytosis of dead NETs by monocytes/macrophages results in intracellular degradation of CD14, which is expressed highly in NETs, and the production of a presepsin, which is released into the extracellular space. 